\title{
Cellular Automaton Simulation for the Oxidation of Binary Alloys
}

\author{
Weigang $\mathrm{Xu}^{1}$, Houzhang $\operatorname{Tan}^{1, *}$, Yuanyi Liu ${ }^{1}$, Yibin Wang ${ }^{1}$ and Shien Hui ${ }^{1}$ \\ ${ }^{1}$ MOE Key Laboratory of Thermo-Fluid Science and Engineering, Xi'an Jiaotong University, Xi'an 710049, China
}

\begin{abstract}
In this study, a cellular automaton model for the oxidation of binary alloys is proposed, by treating internal and external oxidation separately and simultaneously. As an example, the oxidation of nickel-chromium alloys with the formation of spinel and the doping effect is simulated. The oxidation rate and the micro-morphology of simulation are compared with experimental results of others to verify the reliability of the model. The influences of alloy content, reaction temperature and interdiffusion velocity of alloys on the oxidation rate are forecast. Furthermore, the model is capable to simulate the oxidation in oxidants other than oxygen.
\end{abstract}

\section{Introduction}

N. Birks [1] has presented a categorization of simple types of alloy oxidation in which alloys will be grouped as (a) noble parent with base alloying elements and (b) base parent with base alloying elements. The group (a) includes alloy bases, such as aurum, silver, platinum, etc., which do not form stable oxides under normal conditions with alloying elements such as copper, nickel, iron, cobalt, chromium, etc., which form stable oxides. A random-walk cellular automaton model of oxide precipitation in alloys has been developed by Zhou $\mathrm{L}$ et al $[2,3]$, to simulate internal oxidation and its transition to external oxidation. The model has also been used to simulate the phenomena of internal nitridation [4-6]. The group (b) consists of a base of nickel, iron, cobalt, etc., which forms a moderately stable oxide, and an alloying element chromium, aluminum, or silicon, which forms a highly stable oxide. It is generally much more complex as a result of the interaction between internal oxidation and external oxidation.

In this study, a cellular automaton model for oxidation of binary alloys is proposed. The model describes that ionic transport processes through the oxide are accompanied by ionizing phase-boundary reactions and formation of new oxide at a site whose position depends upon whether cations or anions are transported through the oxide layer. For oxidation of binary alloys, the internal oxidation and the solid-state reaction between internal oxides and external oxides are considered. In present model, the migration of oxides in metals is neglected, while the dissociation of internal oxides is considered.

As an example, the oxidation of nickel-chromium alloys at high oxygen partial pressures are simulated to forecast the dependence of the oxidation rates on the reaction temperature, the alloy content, and the alloy interdiffusion velocity. The reliability of the model is verified by comparing the oxidation rate and the micromorphology of simulation with previous experimental results of others.

\section{Model and Simulation Program}

Extensive studies of the oxidation behavior of nickelchromium alloys have been published by a number of investigators [7-9]. Alloys in this system with low chromium contents show internal oxidation of chromium forming chromium hemitrioxide islands within a matrix of almost pure nickel. An outer scale of nickel oxide is formed with an inner layer of nickel oxide containing $\mathrm{NiCr}_{2} \mathrm{O}_{4}$ islands as shown in Figure 1.

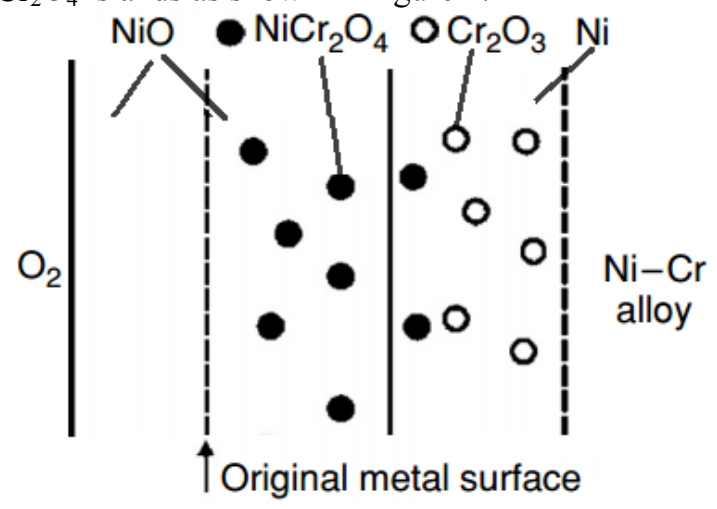

Figure 1. Schematic diagram of the oxidation morphology of dilute $\mathrm{Ni}-\mathrm{Cr}$ alloys

\subsection{Cellular space}

Cellular automata refer to an idealization of physical systems where space, time and physical state are discrete [10]. The space is divided into uniform cells. Each cell may have a finite number of states, and its state may change according to its neighborhood at every step of time.

The system we modeled contains three overlapping two-dimensional square lattices. Each cell of the first lattice is occupied by six states and, in other words, occupied by six species. These are: the base metal A (nickel), the alloying element $\mathrm{B}$ (chromium), three oxides $\mathrm{AO}$ (nickel oxide), $\mathrm{BO}$ (chromium hemitrioxide), $\mathrm{ABO}$ ( $\mathrm{NiCr} 2 \mathrm{O} 4$ spinel) and the gas phase $\mathrm{G}$. The oxygen atoms $\mathrm{O}$ and nickel vacancies VA occupy other two lattices which denote the interstitial and vacancy sites.

\subsection{Simulation of Diffusion}

Chopard and Droz [10] have described a model for the simulation of atomic diffusion. In every cell only a finite number of atoms can be present. The atoms are given a certain direction of motion. By entering the neighboring 
cell, the atoms may change their directions. Thus, each atom selects, at random, a new velocity among the possible values permitted by the lattice, and the new direction is derived from the original direction by random rotation. The random motion is obtained by permuting the direction of the incoming atom. The rotations are weighted with distinct probabilities pl, whereby the probability for clockwise and counterclockwise deflection is assumed to be equal. Thus, the probabilities $\mathrm{p} 0$ for ballistic movement, $\mathrm{p} 2$ for reflection and $\mathrm{p}$ for deflection satisfy $p 0+2 p+p 2=1$. As described in [10], the diffusion coefficient $\mathrm{D}$ can be expressed as

$$
D=\frac{\lambda^{2}}{\tau}\left(\frac{1}{2-2 p_{0}+2 p_{2}}-\frac{1}{4}\right)
$$

Here, $\lambda$ and $\tau$ are parameters of cell size and time step. By varying the probabilities of different atoms in different species, every diffusion coefficient can be obtained.

The interdiffusion of alloys is described as random exchanges of metal cells and determined by the parameter s. While $\mathrm{O}$ diffusion proceeds with every iteration step, the interdiffusion of alloys is considered to occur s times.

The oxygen atoms in the gas phase $G$ and in the metals at the scale-metal interface are replenished at every step of time, to maintain the oxygen partial pressure at interface.

\subsection{Simulation of Reaction}

\subsubsection{Rules of External Oxidation}

Nickel oxide is a p-type cation-deficit semiconductor and, therefore, the cations will migrate with electrons from the scale-metal interface to the scale-gas interface during oxidation[1]. Correspondingly, there will be a flow of defects, cation vacancies and electron holes, in the opposite direction. Consequently, the driving force for the reaction will be reflected by the concentration gradient of cation vacancies across the scale. At high oxygen partial pressures, the oxygen is fully ionized forming another hole and a nickel ion enters the surface to partner the oxygen, thus forming a vacancy in the cation sub-lattice and an extra unit of nickel oxide on the surface of the oxide. The process can be represented by Equation (2),

$$
\frac{1}{2} \mathrm{O}_{2}=\mathrm{O}_{\mathrm{O}}+\mathrm{V}_{\mathrm{Ni}}^{\prime \prime}+2 \mathrm{~h}
$$

At the scale-metal interface the nickel vacancies are filled with metal and pores are left. In present model, the formation of pores and the diffusion of electrons are neglected. Considering that Pilling-Bedworth ratio (PBR) of nickel oxide is greater than 1 , this reactions can be described by the following approach: two parts of nickel oxide are simultaneously formed at the scale-metal interface and the scale-gas interface, which can be understood as the pores are filled because of the plastic deformation of the new oxide film.
At the scale-metal interface, 1 cell of $\mathrm{AO}$ is formed and replaces the cell of A according to Equation (3)

$$
\mathrm{VA}+\mathrm{A}=\mathrm{AO}
$$

At the same time, (a-1) cell of AO is formed at the scale-gas interface according to Equation (4)

$$
\mathrm{O}=(a-1) \mathrm{AO}+\mathrm{VA}
$$

The total reaction is

$$
\mathrm{A}+\mathrm{O}=a \mathrm{AO}
$$

Here, a denotes the Pilling-Bedworth ratio of AO.

\subsubsection{Rules of Internal Oxidation}

The simulation of internal oxidation follows a different cellular automata approach, as proposed in [2, 3]. Because of the external oxidation, the influence of $\mathrm{AO}$ should also be considered. As BO is highly stabler than $\mathrm{AO}, \mathrm{BO}$ near $\mathrm{AO}$ will not dissociate and $\mathrm{B}$ near $\mathrm{AO}$ will be oxidized. 1:1 type of formula for $\mathrm{B}$ oxide is assumed and volume expansion in internal oxidation is not considered. With every iteration step, each cell's state is evaluated simultaneously according to the following modified transition rules:

(I) State B: If its four interstitial sites contain O, it transforms to $\mathrm{BO}$ and a randomly chosen $\mathrm{O}$ atom disappears. Otherwise, if its four nearest neighbors in the von-Neumann neighborhood contain a cells of $\mathrm{AO}$, it transforms to $\mathrm{BO}$ and a cells of $\mathrm{AO}$ chosen randomly transforms to A.

(II) State BO: If its four nearest neighbors in the vonNeumann neighborhood Contain AO state or its four interstitial sites contain $\mathrm{O}$, no transformation is occurring. If its four nearest neighbors in the von-Neumann neighborhood are all in BO state, no transformation is occurring. If more than 1 of its eight neighboring cells of the Moore neighborhood are in BO state and can form together with it at least one compact 4-cell square block of BO state, it will decompose to B state and release a free $\mathrm{O}$ atom at a possibility of $\mathrm{pT}$. In all other cases, it decomposes at a transition probability of pTI whereby $\mathrm{pTI}=\mathrm{pT} 1 / \mathrm{r}$. The setting of parameter $\mathrm{r}$, which determines the correlation between $\mathrm{pT}$ and $\mathrm{pTI}$, is discussed in more detail in [2].

\subsubsection{Combination Rules of External Oxidation and Internal Oxidation}

As the outer scale advances into the metal, the internal oxide islands of chromium hemitrioxide are engulfed by nickel oxide and a solid-state reaction ensues to form NiCr2O4:

$$
\mathrm{NiO}+\mathrm{Cr}_{2} \mathrm{O}_{3}=\mathrm{NiCr}_{2} \mathrm{O}_{4}
$$

The nickel oxide of the inner duplex scale layer contains chromium ions in solution in equilibrium with the NiCr2O4 second phase. This provides cation vacancies: 


$$
\mathrm{Cr}_{2} \mathrm{O}_{3} \stackrel{\mathrm{NiO}}{\longrightarrow} 2 \mathrm{Cr}_{\mathrm{Ni}}^{\circ}+\mathrm{V}_{\mathrm{Ni}}^{\prime \prime}+3 \mathrm{O}_{\mathrm{O}}
$$

The transition rules can be described as:

$$
2 \mathrm{BO}+\mathrm{aAO}=(\mathrm{a}+2) \mathrm{ABO}+\mathrm{VA}
$$

\section{RESULTS AND DISCUSSIONS}

An example of the oxidation of nickel-chromium alloys at $1000{ }^{\circ} \mathrm{C}$ in 1 atm oxygen is simulated with 3000 iteration steps and $\tau=6 \mathrm{~s}$. The square lattices are $200 \times 200$ with $\lambda=1 \mu \mathrm{m}$. The diffusion coefficient of the nickel vacancies in nickel oxide is $3 \times 10-7 \mathrm{~cm} 2 / \mathrm{s}$, and the diffusion of the nickel vacancies in other species and the interdiffusion of alloys are neglected. The mole fraction of the $\mathrm{O}$ at the scale-metal interface is 0.1 and the diffusion coefficient of the $\mathrm{O}$ in metals is $1 \times 10-13 \mathrm{~cm} 2 / \mathrm{s}$. The Pilling-Bedworth ratio of nickel oxide is considered as 2 for simplification.

The Micro-morphologies of nickel-chromium alloys oxidized for $5 \mathrm{~h}$ are given in Figure 2. The parabolic rate

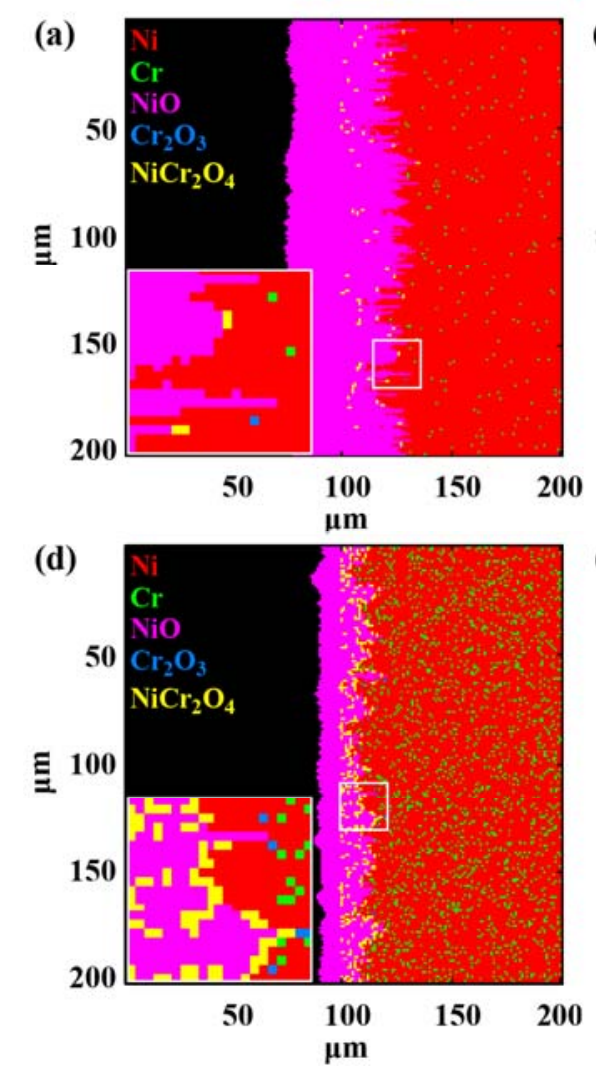

constants are shown in Figure 3. It becomes obvious that the simulation parabolic rate constants match with previous experimental results [1, 7-9]. The differences may be due to the Pilling-Bedworth ratio of nickel oxide changed from 1.78 to 2 or other model simplifications.

As shown in Figure 2(a), (b), and Figure 3, with low chromium content, the doping effect results in an increase in the oxidation rate constant, compared with pure nickel, which is also increased, on a mass-gain basis, due to the extra oxygen tied up in chromium hemitrioxide in the internal oxidation zone.

Since cation diffusion through the $\mathrm{NiCr} 2 \mathrm{O} 4$ spinel is much slower than through nickel oxide, the spinel islands in the scale block the diffusion of the outward migrating nickel ions. Consequently, as the chromium content of the alloy is increased, the increasing volume fraction of spinel reduces the total nickel flux through the scale and the rate constant begins to fall. This is illustrated in Figure 2(c), (d) and Figure 3.
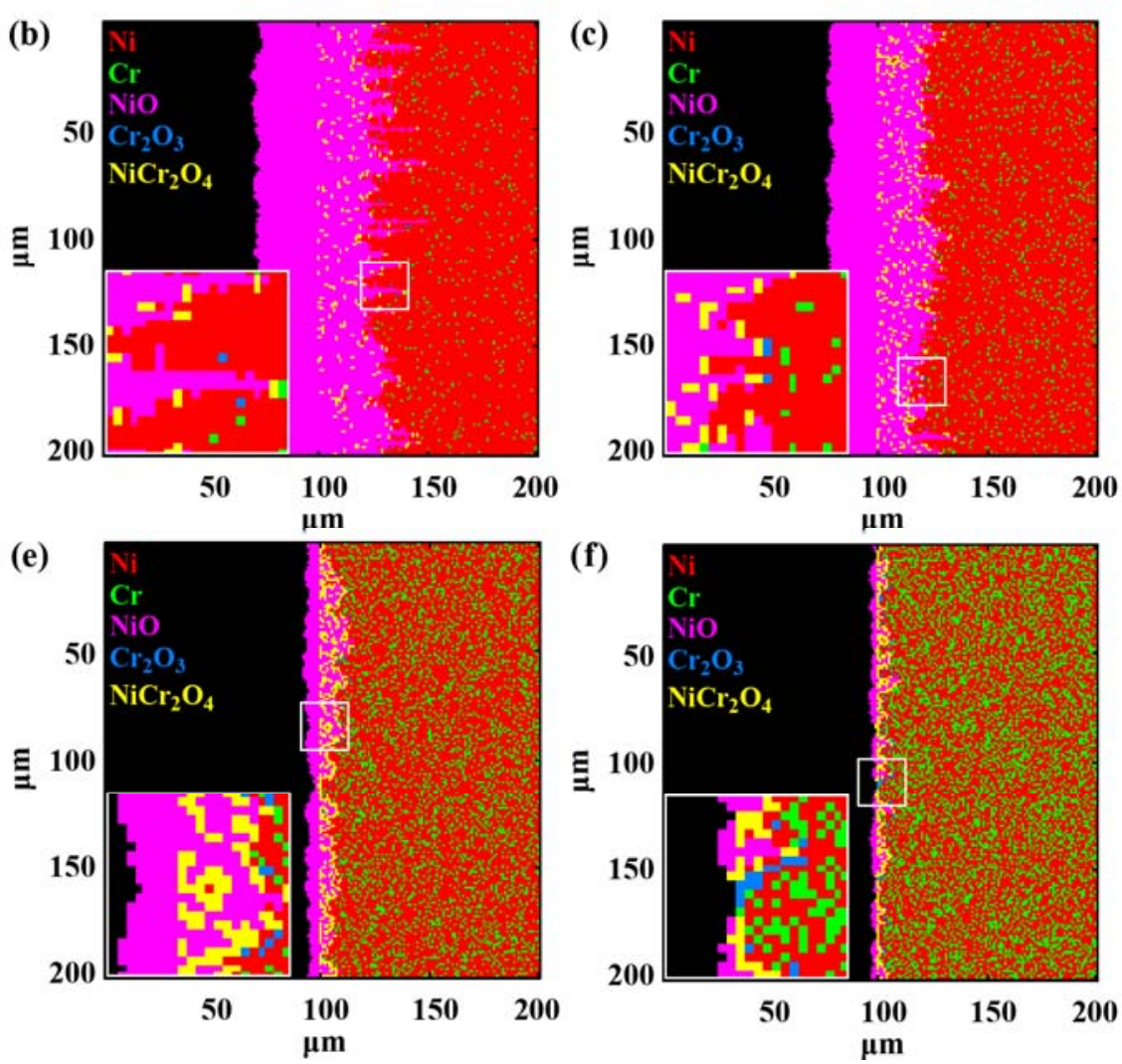

Figure 2. Micro-morphologies of nickel-chromium alloys oxidized for $5 \mathrm{~h}$ at $1000{ }^{\circ} \mathrm{C}$ in 1 atm oxygen: (a) $\mathrm{Ni}-1 \mathrm{wt} \% \mathrm{Cr}$; (b) Ni-2 wt $\%$ Cr; (c) Ni-6 wt $\%$ Cr; (d) Ni-15 wt $\%$ Cr; (e) Ni-20 wt $\%$ Cr; (f) Ni-30 wt $\%$ Cr. 


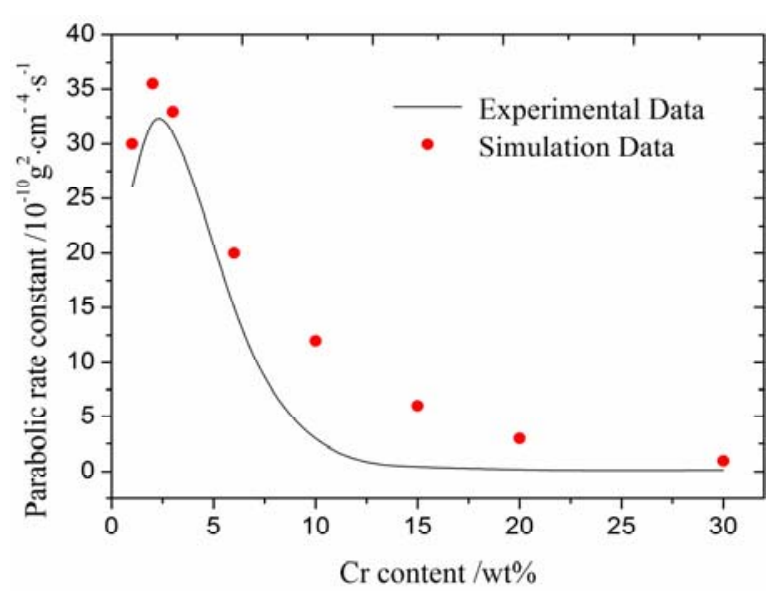

Figure 3. Parabolic rate constants of the oxidation of nickelchromium alloys at $1000^{\circ} \mathrm{C}$ in $1 \mathrm{~atm}$ oxygen

Eventually as the chromium content is increased to about $20 \mathrm{wt} \%$ at $1000{ }^{\circ} \mathrm{C}$, the mode of oxidation changes to give a complete external scale of chromium hemitrioxide, as shown in Figure 2(e) and (f). At this and higher chromium contents the oxidation rate falls abruptly to values more typical of chromium than of nickel, as illustrated in Figure 3.

Figure 4 shows the dependence of the oxidation rate for nickel $-3 \mathrm{wt} \%$ chromium Alloys on temperature. The diffusion coefficients of the nickel vacancies in nickel oxide at $1000{ }^{\circ} \mathrm{C}, 900{ }^{\circ} \mathrm{C}$ and $800{ }^{\circ} \mathrm{C}$ are considered as $3 \times 10-7 \mathrm{~cm} 2 / \mathrm{s}, \quad 1 \times 10-7 \mathrm{~cm} 2 / \mathrm{s}$ and $3 \times 10-8 \mathrm{~cm} 2 / \mathrm{s}$. Lowering the reaction temperature, will decrease the diffusion coefficient and decrease the reaction rate.

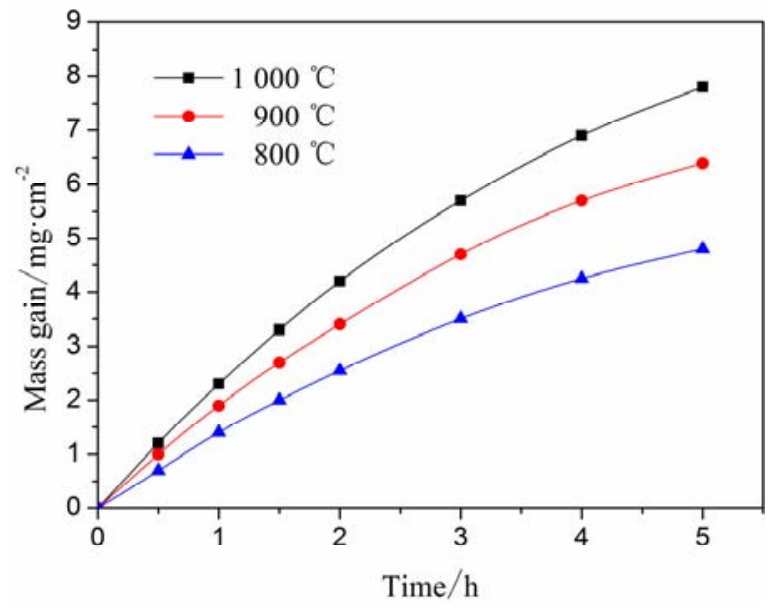

Figure 4. Dependence of the oxidation rate for nickel-3 $\mathrm{wt} \%$ chromium Alloys on temperature in $1 \mathrm{~atm}$ oxygen

The influence of the interdiffusion of alloys is shown in Figure 5. The critical content of the alloying elements to form the protective scale can be reduced by increasing the interdiffusion velocity of alloys. At this point, $\mathrm{O}$ diffusion into the alloy is blocked and a transition to outer layer formation is occurring. This is in accordance with experimental observations, where protective scale formation depends on the concentration and the ratio of diffusivities of the solute and the penetrating element.

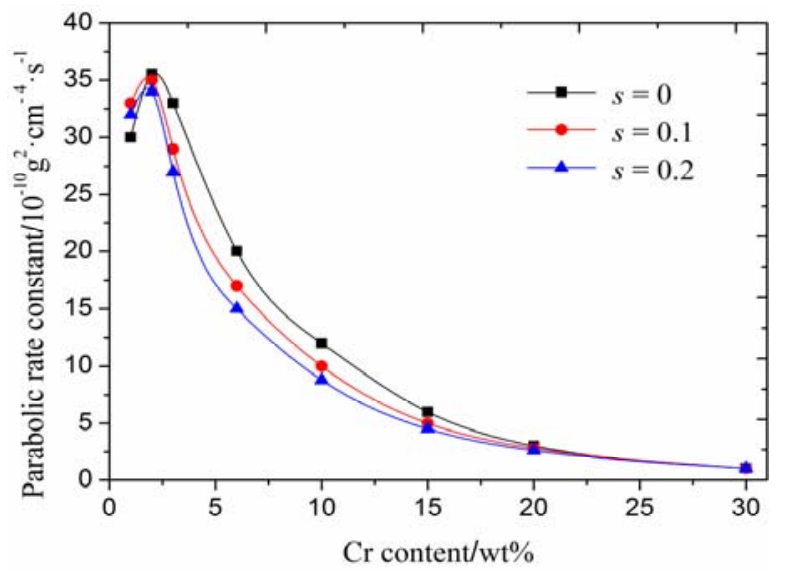

Figure 5. Dependence of the parabolic rate constant for the oxidation of nickel-chromium alloys on interdiffusion velocity of alloys in $1 \mathrm{~atm}$ oxygen at $1000^{\circ} \mathrm{C}$

\section{CONCLUSIONS}

In summary, we develop a new numerical tool based on the cellular automata approach to simulate oxidation phenomena of binary alloys with the formation of two or more oxides. A kinetic model of internal oxidation and external oxidation, coupling random walk diffusion and probabilistic cellular automaton growth, has been developed and programmed for computer simulation in two dimensions. Simulated experiments of oxidation processes of nickel-chromium alloys have been carried out with respect to the effects of reaction temperature, alloy content, and alloy interdiffusion velocity. The results show reasonable agreement with experimental data and theoretical analysis. As shown in this study, it is possible to describe oxidation phenomena of binary alloys for simple systems. The model also can be used to simulate the oxidation in oxidants other than oxygen.

\section{References}

[1] N. Birks, G.H. Meier, F.S. Pettit, Introduction to the High Temperature Oxidation of Metals, second ed., (Cambridge University Press, New York, 2009)

[2] L. Zhou, X. Wei, Scripta Mater., 37, 1483-1489 (1997)

[3] L. Zhou, X.Q. Wei, Scripta Mater., 40, 365-374 (1999)

[4] L. Zhou, X.Q. Wei, Z.M. Deng, Comp. Mater. Sci., 23, 204-208 (2002)

[5] K. Jahns, M. Landwehr, J. Wubbelmann, U. Krupp, Oxid. Met., 79, 107-120 (2013)

[6] K. Jahns, M. Landwehr, J. Wubbelmann, U. Krupp, Mater. Corros., 65, 305-311 (2014)

[7] N. Birks, H. Rickert, J. I. Met., 91, 308-310 (1963)

[8] C.S. Giggins, F.S. Pettit, T. Metall. Soc. Aime, 245, 2509-2514 (1969)

[9] C.S. Giggins, F.S. Pettit, T. Metall. Soc. Aime, 245, 2495-2502 (1969)

[10]B. Chopard, M. Droz, Cellular Automata Modeling of Physical Systems, (Cambridge University Press, 1998) 